\title{
Complications of ileal conduit diversion in adults with cancer followed up for at least five years
}

\author{
DAVID E NEAL
}

\begin{abstract}
A total of 111 adults with malignant disease of the bladder were studied to determine the long term complications of ileal conduit diversion. Each patient had survived at least five years (mean 10 years) after cystectomy.

At final follow up the radiological appearance of one or both kidneys had deteriorated in $50(47 \%)$ of 107 patients: deterioration worsened significantly $(p<0.01)$ with increasing duration of follow up. Eighteen patients (16\%) developed biochemical evidence of impaired renal function, of whom four died of complications of renal failure. Bilateral upper tract dilatation was noted in 30 patients (28\%), and in 21 its cause was obscure. Ten patients formed renal stones, and an additional 12 required further operations on the conduit or stoma.

Despite the age of patients with bladder cancer and the poor prognosis of those with invasive tumours clinically important side effects were observed in a significant proportion of the long term survivors. Further efforts to determine the aetiology of upper tract dilatation in patients with an ileal conduit diversion are justified.
\end{abstract}

\section{Introduction}

The long term results of ileal conduit diversion in children are poor. Of particular concern is the deleterious effect of ileal conduit diversion on the upper urinary tract. This complication has been observed in just under a third of children after operation, ${ }^{1-4}$ and the overall long term complication rate renders ileal conduit diversion unsatisfactory in the treatment of

\footnotetext{
Department of Urology and University Department of Surgery, Department of Urology, Freeman Hospital, Newcastle upon Tyne NE7 7DN

DAVID E NEAL, MS, FRCS, first assistant
}

children with neurogenic bladder dysfunction. ${ }^{14}$ Nowadays ileal conduit diversion is also being used less often in adults with neurogenic bladder dysfunction.

Most patients with invasive bladder cancer have systemic metastases when they present. For them effective palliation and a good quality of life should be the aim of treatment. Because of their age it is possible that few patients with bladder cancer will survive long enough to develop the long term complications of ileal conduit diversion even if they are cured of their cancer. For these reasons, and because effective treatments other than cystectomy or radiotherapy have not been available, the long term complications of ileal conduit diversion in patients with bladder cancer have not been studied specifically.

New methods of treatment for invasive bladder cancer have recently been described. ${ }^{5}{ }^{6}$ The initial results of radical transurethral resection of the tumour or partial cystectomy plus systemic chemotherapy suggest that adequate control of local disease may be achieved in selected patients. ${ }^{56}$ In view of these observations and the paucity of other studies I have examined the long term results of ileal conduit diversion in patients with bladder cancer.

\section{Patients and methods}

Only patients with ileal conduit diversion who had survived more than five years after cystectomy were studied. Patients were followed up regularly in a stoma clinic. Intravenous urography was carried out biennially and serum creatinine and urea and electrolyte concentrations measured at each visit; creatinine was not measured routinely before 1973. Radiological examination of the conduit (loopogram) was not performed routinely.

I studied 111 patients (34 women, $77 \mathrm{men}$ ). Each had undergone cystectomy and ileal conduit diversion for pelvic malignancy and each had survived at least five years (mean $10(1 \mathrm{SD}=3)$ years; range 5-18). Their age at operation ranged from 37 to 74 years (mean 58 (SD 8) years). Of the 111 patients, 66 had received preoperative radiotherapy (dose equivalent to 45 Gy (4500 rads)), cystectomy being carried out between six and eight weeks later. A total of 105 patients had bladder cancer ( 53 invasive, 52 superficial), of whom 94 had transitional cell carcinoma, seven squamous carcinoma, and four adenocarcinoma. Five of the 111 had locally advanced carcinoma of the cervix and the remaining patient had leucoplakia of the bladder. 
Surgical technique-In 49 patients the ureteroileal anastomosis had been fashioned by techniques described by Wallace. ${ }^{78}$ Forty four patients had undergone an "antireflux" type of end to side anastomosis, ${ }^{\circ}$ the remaining 18 simple end to side anastomosis.

Radiological appearances of the upper tract were graded as 0 , normal; 1 , dilated ureter; 2 , mild hydronephrosis; 3 , moderate/severe hydronephrosis but no loss of parenchyma; 4, hydronephrosis plus loss of parenchyma; and 5, non-functioning kidney.

Biochemical impairment of renal function-Serum creatinine concentration does not increase significantly with age in adults. ${ }^{10}$ Impairment of renal function was therefore defined as a serum creatinine concentration of more than $140 \mu \mathrm{mol} / 1(1.6 \mathrm{mg} / 100 \mathrm{ml})$ or an increase in serum creatinine of more than $50 \mu \mathrm{mol} / 1(0.6 \mathrm{mg} /$ $100 \mathrm{ml}$; when measured on at least two consecutive occasions).

Statistical analysis-Results are quoted as means and one standard deviation (SD). Significance was assessed by the Mann-Whitney $U$ test, the Wilcoxon test, or the $\chi^{2}$ test with correction for continuity.

\section{Results}

Recurrent neoplasm-Nine of the 111 patients developed recurrent malignancy of urothelial origin more than five years after operation eight of them died. Two of the nine developed tumours of the renal pelvis and were excluded from the biochemical or radiological assessment of renal function after operation.

Renal failure-Eighteen patients $(16 \%)$ who had normal renal function before operation developed biochemical evidence of impaired renal function in the long term after diversion. Four of the 18 died of complications related to renal failure. Dilatation of the upper tract was noted in 14 of the 18 patients but a cause for the dilatation could be assigned in only four. Serum creatinine concentration at final follow up (117 (SD 57) $\mu \mathrm{mol} / 1 ; 1.3(0 \cdot 6) \mathrm{mg} / 100 \mathrm{ml})$ was no significantly greater than before operation $(95(16) \mu \mathrm{mol} / 1 ; 1 \cdot 1(0 \cdot 2)$ $\mathrm{mg} / 100 \mathrm{ml}$ ) (table). A small but statistically significant increase in creatinine concentration, however, was documented in those patients in whom paired measurements of creatinine were available before and after operation (before operation 95 (SD 16) $\mu \mathrm{mol} / 1(1 \cdot 1(0 \cdot 2) \mathrm{mg}$ $100 \mathrm{ml})$; at five years $102(27) \mu \mathrm{mol} / 1(1.2(0.3) \mathrm{mg} / 100 \mathrm{ml})$; at eight years 109 (34) $\mu \mathrm{mol} / 1(1.2(0.4) \mathrm{mg} / 100 \mathrm{ml}) ; \mathrm{p}<0.01)$, although when patients who developed renal impairment (defined above) were excluded no significant increase in creatinine was observed in the remainder.

Mean ( $S D)$ serum creatinine concentrations ( $\mu \mathrm{mol} / \mathrm{l}$ ) measured before operation, at final follow $u p$, and $5,8,11$, and 14 years after operation

\begin{tabular}{cccccc}
\hline Before operation & Final follow up & 5 years & 8 years & 11 years & 14 years \\
\hline$(n=62)$ & $(n=109)$ & $(n=94)$ & $(n=76)$ & $(n=47)$ & $(n=25)$ \\
$95(16)$ & $117(57)$ & $100(28)$ & $108(32)$ & $112(34)$ & $125(44)$
\end{tabular}

Values after operation compared with before not significantly different.

Conversion: SI to traditional units-Creatinine: $1 \mu \mathrm{mol} / 1 \approx 0.01 \mathrm{mg} / 100 \mathrm{ml}$.

Assessment of upper tract (intravenous urography)-Four of the 111 patients were excluded from assessment of radiological findings (two had renal tumours, two had an incomplete examination). The results of radiological assessment of 214 renal units were therefore available. Before diversion 177 renal units $(83 \%$ ) had been normal and 37 had been abnormal: three patients had a non-functioning kidney due to obstruction, 18 had unilateral upper tract dilatation, and eight had bilateral upper tract dilatation. After diversion deterioration in the appearance of one or both kidneys occurred in $50(47 \%)$ of the patients. When the results of radiological examination of all 214 units (whether normal or abnormal before operation) were assessed (fig) deterioration was found to worsen with increasing duration of follow up: a significant deterioration in the appearance of the upper tract occurred beiween five and 11 years after operation $\left(\chi^{2}=6.9 ; p<0.01\right)$. In addition, a significant increase in the severity of upper tract abnormality (from grades 1 and 2 to grades 3 and 4 ) occurred over the same period $\left(\chi^{2}=12.4 ; p<0.001\right)$. No systematic difference was observed when renal units that had been normal before operation were compared with those that had been abnormal before operation. Nevertheless, five years after operation the incidence of severe abnormality (grade 3 or worse) was higher in units that had been abnormal before operation (14/37) than in units that had been normal before operation $\left(18 / 177 ; \chi^{2}=16.3 ; p<0.001\right)$. Radiotherapy did not appear to have a deleterious effect on the long term results. No significant differences were observed when patients who had received radiotherapy before operation were compared with those who had not. Of the 37 renal units that were abnormal before diversion, $15(41 \%)$ returned to normal within the first year, 19 were unchanged or became more dilated, and the three non-functioning kidneys became small and atrophic.

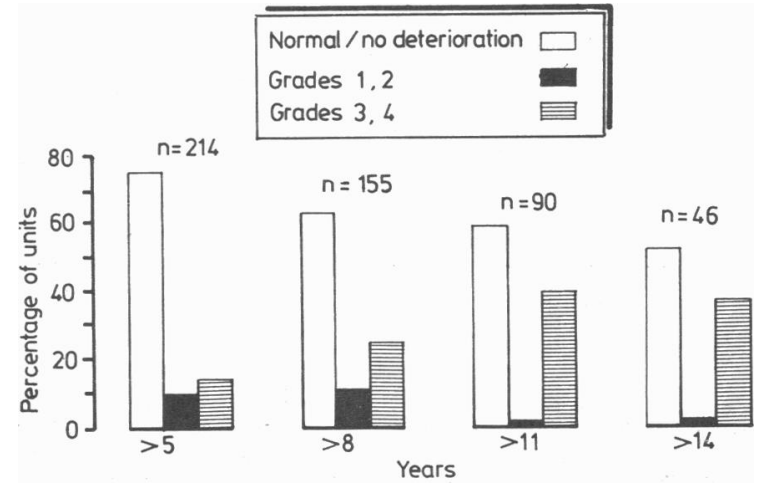

Effect of ileal conduit diversion on 214 upper tracts. Chart shows proportion of renal units that were normal or abnormal, and degree of abnormality, $5,8,11$, and 14 years after operation.

Assessment of conduit (loopogram) - A total of 130 renal units were studied by retrograde injection of contrast along the conduit. Since patients with a normal intravenous urogram were not routinely subjected to loopograms, only 47 of the 130 units were normal: free reflux was observed in 34 of them $(72 \%)$. The remaining 83 units were dilated: 77 showed free reflux $(93 \%)$ and six no reflux. The absence of reflux was considered to be due to stricturing in three, kinking of the anastomosis in two, and narrowing by the sigmoid mesocolon in one. No significant benefit was observed from the use of an antireflux anastomosis compared with a freely refluxing anastomosis. Thus the rates of reflux after the two types of operation $(81 \% v 89 \%)$ and the incidences of upper tract dilatation $(59 \% v 68 \%)$ were similar.

Bilateral dilatation of the upper tract was observed in 30 patients $(28 \%)$. A satisfactory explanation was found in the conduit or at the ureteroileal anastomosis in only nine. Grade 3 or 4 dilatation was present in 14 of the remaining 21 patients and biochemical evidence of renal impairment was found in 10 of the 21 .

Reoperation-Twelve patients had a further operation on the stoma or conduit. Reoperation was performed for prolapse or retraction $(n=3)$, stomal stenosis $(n=7)$, or stricture formation within the conduit $(n=2)$. Revision of the ureteroileal anastomosis for stricture was carried out in three patients. Seven patients developed a nonfunctioning kidney, of whom four underwent nephrectomy. Ten additional patients developed renal stones: four underwent nephrolithotomy and two nephrectomy.

\section{Discussion}

The incidence of complications observed in these patients with malignant disease who had survived at least five years after cystectomy and ileal conduit diversion should cause concern, particularly the finding that at final follow up only 57 of 107 patients had upper tracts that had not deteriorated.

Poor long term results have been well described in children subjected to ileal conduit diversion. ${ }^{1-4} 11$ It is disappointing, but perhaps not surprising, that adults with malignant disease also have poor long term results. Others who have studied adults with a wide variety of underlying diseases have found a lower incidence of upper tract abnormalities. ${ }^{212}$ Indeed, some have even recorded overall improvement in the appearance of the kidneys after diversion. ${ }^{13}$ This discrepancy is probably attributable to the relatively short length of follow up in some of the studies and the heterogeneous nature of the groups 
studied. Poor results after surgery may be related to individual surgical technique, and Graham has reported good results in a personal series of children subjected to ileal conduit diversion. ${ }^{14}$ Moreover, although dilatation of the upper tract may arise years after operation, technical factors predisposing to ischaemia may be relevant in the aetiology of functionally important obstruction of the distal conduit. ${ }^{15}$

The incidence of bilateral upper tract dilatation noted in this study was similar to that reported in studies in children. ${ }^{1411}$ Scott commented that dilatation was not due to obvious obstruction, for many patients appeared to have wide, healthy stomas and normal loopograms. ${ }^{1}$ Ureteroileal reflux cannot of itself be the cause of dilatation, since many patients with reflux have normal upper tracts. A combination of high pressure in the conduit and reflux across the ureteroileal anastomosis may explain the dilatation.

Many children and young adults with non-malignant disease who might be considered for a urinary diversion should have a good life expectancy. It is now accepted that because of its long term morbidity diversion should be avoided whenever possible in these patients. On the other hand, because so few patients with bladder cancer will survive to develop long term side effects the use of ileal conduit diversion might be thought acceptable in them. Nevertheless, clinically important complications were observed in the patients in this study. In particular, the finding that bilateral upper tract dilatation in the absence of an obvious cause is commonplace and that it may be associated with renal damage signifies that further efforts should be made to determine its cause.
I thank Sister A Lawson, $\mathrm{Mr}$ W K Yeates, $\mathrm{Mr} \mathrm{D} M$ Essenshigh, Mr R R Hall, and Professor J Swinney, whose careful records permitted this study to be carried out. Thanks are also due to Mr R R Hall and Mr P D Ramsden for constructive criticism of the manuscript. I thank the William Leech Property Trust and the Northern Counties Kidney Research Fund for financial support.

\section{References}

1 Scott JES. Urinary diversion in children. Arch Dis Child 1973;48:199-206 Pitts WR, Muecke EC. A 20 year experience with ileal conduits: the fate of the kidneys. F Urol $1979 ; 122: 154-7$.

PJB, Slade $\mathrm{N}$. The long term results of ileal conduit urinary diversion in children. Br $\mathcal{f}$ Urol $1979 ; 51: 458-61$.

Middleton AW, Hendren WH. Ileal conduits in children at the Massachuset General Hospital from 1955 to 1970 . F Urol 1976;115:591-5.

Socquet $Y$. Combined surgery and adjuvant chemotherapy with high dose methotrexate and folinic acid rescue (HDMTX-CF) for infiltrating tumour of the bladder. $\mathrm{Br} F$ Urol $1981 ; 53: 439-43$.

6 Hall RR, Newling DWW, Ramsden PD, Richards B, Robinson MRG, Smith $\mathrm{PH}$. Treatment of invasive bladder cancer by local resection and high dose

7 Wallace DM. Ureteric diversion using a conduit. A simplified technique. $\mathrm{Br} \mathfrak{F}$ Urol 1966;38:522-7.

Wallace DM. Uretero-ileostomy. Br f Urol 1970;42:529-34.

Leadbetter WF, Clarke BG. Five years' experience of uretero-enterostomy with

Payne RB. The assessment of glomerular function:

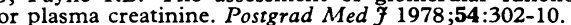
creatinine clearance or plasma creatinine. Postgrad $\mathrm{Med}$ in children: compute analysis of follow up from 2 to 16 years. $\mathcal{F}$ Urol $1975 ; 114: 285-8$.

12 Philp NH, Williams JL, Byers CE. Ileal conduit urinary diversion: long-term follow-up in adults. Br $\mathcal{F}$ Urol $1980 ; 52: 513-9$.

13 Kafetsioulis A, Swinney J. Urinary diversion by ileal conduit. A long-term

14 Graham AG. Long-term results of ileal conduit diversion in children. $\mathrm{Br} f \mathrm{Urol}$

15 McEwan AB, Clark PB. The stoma of the ileal conduit. Br $\mathcal{f}$ Urol $1973 ; 45$ :

(Accepted 28 February 1985)

\begin{abstract}
Of 17032 women taking part in the Oxford Family Planning Association contraceptive study, 4104 stopped using a birth control method to plan a pregnancy on a total of 6199 occasions. The influence of various factors on fertility in these women was assessed by measuring the time taken to give birth to a child. An appreciable inverse relation was observed between age at stopping contraception and fertility both in nulliparous and parous women, but the effect was much greater in the nulliparous women.

The most important finding was a consistent and highly significant trend of decreasing fertility with

\footnotetext{
Department of Community Medicine and General Practice, Radcliffe Infirmary, Oxford OX2 6HE

GEOFFREY HOWE, PHD, visiting professor

CAROLYN WESTHOFF, MD, Milbank scholar

MARTIN VESSEY, MD, FRCPED, professor

DAVID YEATES, PHD, computer scientist

Correspondence to: Professor M Vessey.
}

increasing numbers of cigarettes smoked per day; it was estimated that five years after stopping contraception $10.7 \%$ of smokers smoking more than 20 cigarettes a day, but only $5.4 \%$ of non-smokers, remained undelivered. Some relation was found between fertility and social class, age at marriage, and a history of gynaecological disease, but weight, height, and Quetelet's index were without noticeable effect.

\section{Introduction}

Factors that may affect a woman's fertility, such as her gynaecological and reproductive history, her age, and her smoking habit, are of both biological interest and practical concern. Opportunities to examine associations between such factors and fertility using the epidemiological approach are rare because of the lack of relevant data for adequately sized cohorts of women observed for sufficiently long periods of time during which they are known to be attempting to become pregnant. The Oxford Family Planning Association contraceptive study, which includes data on over 17000 women of childbearing age observed for an average period of about 11.5 years, provides such an opportunity. ${ }^{1}$ Oral contraceptives, and to a lesser extent intrauterine devices, have previously been reported from this study to impair fertility immediately after their discontinuation, but the effect is relatively short lived. ${ }^{2}{ }^{3}$ Here we describe the effects of various other factors on fertility in this cohort. 